\title{
COMMUTING OPERATOR SOLUTIONS OF ALGEBRAIC EQUATIONS
}

\author{
R. C. RIDDELL AND R. B. INSLEY
}

ABstract. Let $G(w, z)$ be a complex polynomial, and $S$ a bounded operator of scalar type on a complex Banach space, whose spectrum avoids the points $\lambda$ for which $G(\lambda, z)=0$ has multiple roots $z$. The form of a bounded operator $T$ which commutes with $S$ and satisfies $G(S, T)=0$ is established.

1. Introduction. Fix a Banach space $X$ over the complex numbers $C$, and let $B$ denote the Banach algebra of all bounded linear operators on $X$. Given $S \in B$ of scalar type, and given a polynomial in two indeterminates

$$
G(w, z)=a_{n}(w) z^{n}+\cdots+a_{1}(w) z+a_{0}(w) \quad\left(a_{i}(w) \in C[w]\right),
$$

we seek operators $T \in ß$ such that

$$
T \text { commutes with } S \text { and } G(S, T)=0 \text {. }
$$

Denoting the spectrum of $S$ by $\sigma$, we assume:

$$
\text { For each } \lambda \in \sigma \text {, the polynomial } G(\lambda, z) \text { has }
$$$$
n \text { distinct complex roots } t_{1}(\lambda), \cdots, t_{n}(\lambda) \text {. }
$$

We shall establish:

THEOREM. Notation and assumptions as above, there exist $T_{1}, \cdots$, $T_{n} \in B$ satisfying (E), and having the following property: $T \in B$ satisfies (E) if and only if there exist $F_{1}, \cdots, F_{n} \in B$ such that

$$
F_{i} \text { commutes with } S \text {, }
$$

$$
\begin{aligned}
F_{i}^{2} & =F_{i}, \quad F_{i} F_{j}=0 \quad \text { for } i \neq j, \quad \Sigma_{i} F_{i}=I, \\
T & =\Sigma_{i} T_{i} F_{i} .
\end{aligned}
$$

An operator $S$ is of scalar type $[1$, p. 332] if $S$ admits a resolution of the identity $E(\cdot)$, and if moreover $S$ can be recovered from $E(\cdot)$ by integration over $\sigma$ :

$$
S=\int \lambda E(d \lambda)
$$

Received by the editors December 31, 1969 and, in revised form, May 20, 1970. AMS 1969 subject classifications. Primary 4740, 4790.

Key words and phrases. Scalar-type operator, algebraic operator equation. 
In particular, a normal operator on a Hilbert space is of scalar type. In the theorem, $G$ need not be irreducible, but by (A) no repeated factors are permitted in the prime decomposition of $G$. The idempotents $F_{i}$ are not asserted to be values of $E(\cdot)$, and indeed need not be, if $S$ has multiplicity greater than 1 .

Foguel [2] proved this theorem for the special case $G(w, z)=g(z)$ $-w, g$ a complex polynomial, and we imitate his proof. For a given solution $T$ of $(\mathrm{E})$, the main step in constructing the $F_{i}$ is to check that $G_{2}(S, T)$ is invertible, where $G_{2}(w, z)=\partial G(w, z) / \partial z$. In Foguel's case, $G_{2}(w, z)=g^{\prime}(z)$ is independent of $w$, and the existence of $g^{\prime}(T)^{-1}$ follows immediately from (A) and the spectral mapping theorem. The general proof below uses maximal ideals, and we are indebted to the referee for a substantial simplification of our original argument.

2. Proof of the theorem. Let $\mathfrak{T}$ denote the Banach algebra of all essentially bounded measurable complex functions on $\sigma$.

LEMмA 1. There exist $t_{1}, \cdots, t_{n} \in \mathscr{T}$ such that, for each $\lambda \in \sigma$,

(1) $G(\lambda, z)=a_{n}(\lambda) \Pi_{j}\left(z-t_{j}(\lambda)\right)$,

(2) $G_{2}(\lambda, z)=a_{n}(\lambda) \Sigma_{i} \Pi_{j \neq i}\left(z-t_{j}(\lambda)\right)$.

Proof. Let $K$ be an oriented cut in $C$ joining the singularities $\lambda_{1}, \cdots, \lambda_{k}$ of the algebraic function(s) determined by $G$. Then the roots $t_{i}(\lambda)$ can be chosen to be holomorphic in $C-K$. If each $t_{i}$ is analytically continued to $K^{\prime}=K-\left\{\lambda_{1}, \cdots, \lambda_{k}\right\}$ from the left, say, then the extended $t_{i}$ are defined and locally bounded on $C^{\prime}=C$ $-\left\{\lambda_{1}, \cdots, \lambda_{k}\right\}$, have, at worst, jump discontinuities from the right along $K^{\prime}$, and have for values $\left\{t_{i}(\lambda)\right\}$ precisely the set of roots of $G(\lambda, z)$, for each $\lambda \in C^{\prime}$. Assumption (A) provides that $\sigma \subset C^{\prime}$, and in particular that $a_{n}(\lambda) \neq 0$ for $\lambda \in \sigma$; hence restricting the $t_{i}$ to $\sigma$ establishes (1), from which (2) is immediate.

By $\left[1\right.$, Lemma 6 , p. 341], the map $\Re \rightarrow B$ given by $f_{\mapsto} f(S)$ $\equiv \int f(\lambda) E(d \lambda)$ is a continuous algebra homomorphism. Clearly it extends by $z \mapsto z$ to a homomorphism $\mathfrak{T}[z] \rightarrow ß[z]$, which carries (1) and (2) over to relations

(3) $G(S, z)=a_{n}(S) \Pi_{j}\left(z-T_{j}\right)$,

(4) $G_{2}(S, z)=a_{n}(S) \Sigma_{i} \Pi_{j \neq i}\left(z-T_{j}\right)$, in which we have set $T_{j}=t_{j}(S)$. Then each $T_{j}$ commutes with $S$ and, by (3), obeys $G\left(S, T_{j}\right)=0$.

Now suppose that $T$ satisfies (E). Then in particular $T$ must commute with $E(\cdot)\left[1\right.$, Theorem 5, p. 329] and hence with the $T_{j}$, so that $z \mapsto T$ defines a homomorphism $B[z] \rightarrow B$, which carries (3) and (4) to 
(5) $0=G(S, T)=a_{n}(S) \Pi_{j}\left(T-T_{j}\right)$,

(6) $G_{2}(S, T)=a_{n}(S) \Sigma_{i} \Pi_{j \neq i}\left(T-T_{j}\right)$.

LEMма 2. $G_{2}(S, T)^{-1}$ exists in $B$ and commutes with $S$ and $T$.

Proof. Let $\mu: a \rightarrow C$ denote any nonzero continuous homomorphism, where $a \subset \otimes$ is the (commutative) full algebra generated by $E(\cdot)$ and $T\left[1\right.$, p. 342]. Then $\lambda=\mu(S) \in \sigma$, for otherwise $(S-\lambda I)^{-1} \in Q$ by definition of "full algebra", and $I=(S-\lambda I)(S-\lambda I)^{-1}$ would go by $\mu$ to $1=0$. Hence $G(\lambda, \mu(T))=\mu(G(S, T))=0$, and then $\mu\left(G_{2}(S, T)\right)$ $=G_{2}(\lambda, \mu(T)) \neq 0$, by $(\mathrm{A})$. Thus $G_{2}(S, T)$ lies in no maximal ideal of $a$, so is invertible in $a$.

Now for each $i$, set

$$
F_{i}=G_{2}(S, T)^{-1} a_{n}(S) \Pi_{j \neq i}\left(T-T_{j}\right) .
$$

To verify (D), notice that $\Sigma_{i} F_{i}=I$ follows from (6). For $i \neq j, F_{i} F_{j}$ contains each factor $T-T_{k}$ at least once, so vanishes by (5); and then $F_{i}^{2}=F_{i}\left(\Sigma_{j} F_{j}\right)=F_{i}$ follows. Similarly $(7)$ and (5) give $\left(T-T_{i}\right) F_{i}=0$ for each $i$, and summing yields $T=\Sigma_{i} T_{i} F_{i}$, to conclude the "only if" part of the proof.

Conversely, suppose that $F_{1}, \cdots, F_{n}$ obey (D). Then each $F_{i}$ commutes with $E(\cdot)$, hence with each $T_{j}$, and $T=\Sigma_{i} T_{i} F_{i}$ commutes with $S$. Moreover, it follows by induction that $T^{k} F_{i}=T_{i}^{k} F_{i}$, hence that $G(S, T) F_{i}=0$, for each $i$. Summing yields $G(S, T)=0$, to conclude the "if" part.

\section{REFERENCES}

1. N. Dunford, Spectral operators, Pacific J. Math. 4 (1954), 321-354. MR 16, 142.

2. S. R. Foguel, Algebraic functions of normal operators, Israel J. Math. 6 (1968), 199-201. MR 38 \#1550.

University of British Columbia, Vancouver, British Columbia 